Recherches en didactique des langues et des cultures

Les cahiers de l'Acedle

11-2| 2014

Plurilinguisme(s) et entreprise : enjeux didactiques

\title{
Le plurilinguisme au cœur de l'action
}

\section{Anne Morel-Lab}

\section{(2)enEdition}

\section{Journals}

Édition électronique

URL : http://journals.openedition.org/rdlc/1770

DOI : $10.4000 /$ rdlc. 1770

ISSN : 1958-5772

Éditeur

ACEDLE

\section{Référence électronique}

Anne Morel-Lab, « Le plurilinguisme au cœur de l'action », Recherches en didactique des langues et des cultures [En ligne], 11-2 | 2014, mis en ligne le 07 juin 2014, consulté le 19 avril 2019. URL : http:// journals.openedition.org/rdlc/1770 ; DOI : 10.4000/rdlc.1770

Ce document a été généré automatiquement le 19 avril 2019

\section{(†) $\ominus$

Recherches en didactique des langues et des cultures is licensed under a Creative Commons AttributionNonCommercial-NoDerivatives 4.0 International License 


\title{
Le plurilinguisme au cœur de l'action
}

\author{
Anne Morel-Lab
}

\section{Introduction}

$1 \quad$ Le terrain d'étude des phases de construction du site industriel de $\mathrm{KNS}^{1}$ (Konianbo Nickel SA) en Nouvelle-Calédonie offre un angle de vue particulier sur la didactique des langues du fait de la complexité des plurilinguismes qui s'y côtoient. En effet, si l'histoire coloniale de ce territoire insulaire du Pacifique Sud et le rapport diglossique entre les langues d'appartenance et le français constitue, comme l'ont largement démontré les travaux en sociolinguistique menés sur le terrain (Vernaudon \& Fillol, 2009 ; Fillol, 2013; Salaün, 2005, 2013), un paradigme tout à fait significatif d'un plurilinguisme de type colonial $^{2}$, celui-ci est bousculé par le développement de grands projets industriels avec l'anglais comme langue de communication. Ma participation observante sur ce terrain d'étude océanien m'a ainsi permis d'être le témoin actif du télescopage entre les représentations coloniales du plurilinguisme et les formes d'exploitation linguistique se développant dans le monde du travail à l'international du fait de sa tertiarisation. Elle m'a amenée à m'interroger sur la prise en charge didactique des pratiques linguistiques en situation professionnelle.

2 Dans une première partie, je dresserai un rapide état des lieux des derniers travaux en matière de plurilinguisme au travail. Je présenterai ensuite le terrain d'étude et la méthodologie de la recherche. De là, une description fine des activités langagières des agents administratifs attachés à l'un des services du projet permettra de rendre visible les compétences mises en œuvre par ces océaniens dans la réalisation de leurs activités professionnelles. Enfin, je développerai en quoi leurs activités qui participent à la tertiarisation de la main d'œuvre mériteraient d'être davantage reconnues et comment les compétences qu'ils mettent en œuvre pour les réaliser ouvrent de nouvelles perspectives en matière de didactique des langues. 


\section{Le plurilinguisme au travail, un champ disciplinaire en pleine expansion}

3 Le champ de la dynamique des langues ne cesse, depuis le tournant des années 1970, d'ouvrir de nouveaux horizons en sociolinguistique. A l'orée du XXIème siècle avec l'internationalisation des modes de production et le développement massif des mobilités professionnelles, les chercheurs en sciences sociales commencent à explorer les rapports de langues dans le monde du travail. Au-delà des divers axes de recherche en lien avec les disciplines sollicitées, les travaux menés dans cette perspective, et qui ont ainsi choisi de s'écarter de la sphère purement éducative, privilégient des approches pluridisciplinaires mieux adaptées à décrire la complexité des situations. Qu'il s'agisse de politique linguistique, de l'économie des langues ou bien encore de management de l'interculturel, les programmes de recherches, tel le projet Dylan ${ }^{3}$ (2006-2011) ou plus récemment Mime (Mobility and Inclusion in Multilingual Europe) lancé en 2014 par le département de traduction et d'interprétation de l'Université de Génève, ont pour souci d'apporter des réponses à la préoccupation grandissante des institutions européennes et des entreprises internationales à devoir gérer la diversité linguistique, le multiculturalisme et le plurilinguisme qui en découlent. De fait, depuis le tournant des années 2000, les bouleversements liés à l'ouverture des marchés économiques ont fortement impacté le marché linguistique en y introduisant de nouveaux rapports de force entre les langues (liés en partie à la domination grandissante des modèles économiques anglo-américains et à l'anglais international qui les véhiculent). Parallèlement l'accroissement des mobilités, qu'elles soient étudiantes, professionnelles ou liées à des phénomènes de migration plus vastes ${ }^{4}$ ont diversifié le paysage linguistique occidental faisant apparaître dans la sphère sociale la présence de langues qui jusque-là n'avaient pas de visibilité en dehors de leurs frontières linguistiques d'origine. L'ensemble de ces phénomènes ont suscité l'intérêt de chercheurs qui, indépendamment de leur discipline, ont choisi de privilégier une démarche ethnographique dans l'approche des langues. C'est ainsi que la part langagière du travail devient un objet de recherche en ethnographie linguistique, champ disciplinaire à propos duquel Monica Heller et Josiane Boutet, fondatrice du réseau Langage et Travail ${ }^{5}$ précisent :

Ce n'est que vers les années soixante-dix, sous l'influence de J. Gumperz, que l'on voit émerger une sociolinguistique interactionniste, qui cherche surtout à explorer les problèmes de l'inégalité sociale; situé davantage dans le domaine de la linguistique appliquée, ce courant cherche à se donner un profil disciplinaire indépendant, sous le nom d'ethnographie linguistique. (Boutet \& Heller, 2007 : 305)

Dans la même ligne, les travaux de Monica Heller et Alexandre Duchêne s'intéressent au marché linguistique du point de vue des acteurs en étudiant les transformations sociales liées à la tertiarisation des activités de production et aux inégalités sociales qui en découlent.

Mes propres travaux menés en situation professionnelle dans le cadre d'un projet industriel international s'inscrivent dans cette démarche avec la particularité d'être situés en contexte postcolonial. Ici, l'approche ethnographique du terrain océanien est influencée par les rapports de diglossie s'exerçant entre le français, langue officielle et véhiculaire de la Nouvelle-Calédonie, l'anglais, langue de communication du projet et les nombreuses langues d'appartenance du Pacifique. Mon adhérence au terrain m'a ainsi 
permis de mettre en lumière la complexité des pratiques langagières d'acteurs invisibles dans l'organisation qui, par nécessité professionnelle, sont amenés à communiquer dans des langues plus ou moins normées au regard des situations auxquelles ils doivent contribuer de manière fonctionnelle ou pragmatique.

\section{Plurilinguisme et parole d'œuvre}

6 Dans cette deuxième partie, je présenterai la méthodologie de ma recherche et ferai la description des caractéristiques du plurilinguisme d'une catégorie d'agents administratifs engagés sur le projet de KNS. Je montrerai ensuite comment ce plurilinguisme non normé devient parole d'œuvre dans le monde du travail qui l'organise et en contrôle les modes de production.

\section{L'adhérence au terrain}

7 Au sein du projet de KNS, mon activité de formatrice indépendante m'a amenée à travailler en étroite collaboration avec l'équipe du Centre de Coordination des Visas, un service interface dont la mission est de mettre en synergie les impératifs des entrepreneurs internationaux engagés dans la réalisation $\mathrm{du}$ chantier de construction avec ceux des partenaires institutionnels, administratifs et politiques de la Nouvelle-Calédonie. La méthodologie de ma recherche est intrinsèquement liée à cette situation professionnelle. À la fois, interlocutrice, formatrice et référente, j'ai eu en charge la création et la mise en place des outils fonctionnels bilingues anglais/français puisque la langue de ces grands projets est l'anglais international qui associe à un lexique technique très spécifique une stylistique minimaliste tandis que le français est imposé par le contexte francophone et la nécessité d'avoir à communiquer en français avec les autorités locales. Ces documents prennent la forme de procédures, de guides, d'exempliers mis à la disposition des usagers et des agents locaux en charge d'établir les contacts et de suivre le montage des dossiers de demande de visas et permis de travail des employés recrutés à l'international. L'efficacité de ces agents visas jeunes océaniens au parcours scolaire marqué par ce que Marie Salaün nomme "L'école indigène" dans son ouvrage paru en 2005, est directement associée à la manière dont ils utilisent ces outils pour accompagner leurs interlocuteurs non-français dans leurs démarches. En effet, leurs compétences linguistiques en français et en anglais (les deux langues de communication du projet) sont globalement peu normées bien qu'acquises pour l'essentiel dans le cadre scolaire. En outre, elles sont hétérogènes: entre A2 et $\mathrm{C} 1$ du Cecrl (Cadre Européen Commun de Références pour les Langues) en fonction des langues, des individus et de leur littéracie.

\section{Un plurilinguisme de la relation}

8 Au fil des années, j'ai pu observer les pratiques développées par ces agents visas pour pallier leurs difficultés linguistiques en anglais et en français en adaptant les outils proposés à leurs besoins spécifiques. J'ai observé l'élaboration des stratégies de coopération, j'ai suivi le développement des réseaux d'information et d'échanges entre collègues leur permettant de trouver des solutions aux problèmes qui se posaient à eux. J'ai été le témoin de leur prise d'autonomie dans la maîtrise des procédés mis en place 
(pour l'essentiel des courriels). J'ai aussi pu relever leur créativité dans l'élaboration de modes de transmission de l'information alliant au formalisme des contraintes juridiques et organisationnelles, l'informel des registres de langues.

Ces pratiques, plus ou moins conscientisées en fonction des acteurs, relèvent de l'implicite. Elles prennent des formes hybrides et souples, adaptables au rang, au statut, à la culture de leurs interlocuteurs. Usant d'une "mise en mots en situation" (Boutet, 1991) pouvant aller du formel le plus déférent à des formes d'oralité faisant usage de la métaphore, de la répétition, les agents visas n'hésitent pas à mêler les langues, le français pour le langage administratif; l'anglais pour le lexique professionnel et des bribes de langues d'appartenance, les leurs ou celles de leurs interlocuteurs. Leurs modulations lexicales et syntaxiques jouent sur divers registres : l'humour, la familiarité, les marques de respect, des expressions prescriptives. Ce faisant, ils cherchent à établir avec leurs interlocuteurs un lien de confiance reposant sur un mélange d'empathie et d'autorité. Comme le faisait remarquer l'un de ces agents, ces conduites relèvent d'un processus visant à éduquer les requérants de manière à les amener à produire les bons documents en conformité avec le prescrit calédonien et les prérequis du projet en terme de calendrier. C'est ainsi, que la part tangible de leur activité : la production en temps voulu d'un dossier correct, ne permet pas de rendre visible la part fondamentale de leur mission: comprendre et se faire comprendre d'interlocuteurs dont les pratiques langagières, les référents culturels et linguistiques leurs sont très éloignés. Ce savoir-faire global fait de compétences relationnelles, interculturelles et communicationnelles dans les langues de référence, relève de processus d'apprentissage spécifiques qui, aux savoirs scolaires, associent d'autres types de savoirs non académiques lesquels sont révélés en situation et dans l'action.

10 Ceci m'a amené à analyser les pratiques langagières associées à la sphère familiale de ces agents visas et au contexte diglossique de la Nouvelle-Calédonie. J'ai ainsi pu relier leur capacité à faire circuler des langues à leur savoir expérientiel d'océanien plurilingue.

11 A l'image de nombre de jeunes français nés de parents étrangers installés en France, les agents visas du CCV (Centre de Coordination des Visas) sont des enfants de la ville, n'ayant qu'une connaissance très sommaire de leurs langues d'appartenance. Ils déclarent les comprendre mais ne pas pouvoir tenir une conversation suivie. De ce fait, ils ont développé des pratiques plurielles qui en fonction de leurs interlocuteurs : la fratrie, les parents, les grands-parents, les cousins, s'adaptent aux compétences linguistiques des uns et des autres. Ces pratiques vont du tout français à des hybridations qui jouent sur :

- l'alternance codique en introduisant des mots en langue dans une phrase de structure française ;

- le fait de répondre en français à des locuteurs qui s'expriment dans une langue locale qu'ils comprennent ou devinent ;

- le truchement par une ou plusieurs autres langues partagées par tout ou partie des interlocuteurs puisque dans le Pacifique, il n'est pas rare qu'au sein d'un même collectif (y compris familial), plusieurs langues d'appartenance soient utilisées, multipliant les situations plurilingues.

12 Ainsi, les agents visas font preuve d'une sorte de souplesse linguistique qui leur permet de communiquer avec et au-delà des langues, faisant référence en cela à ce qu'Edouard Glissant nomme "l'imaginaire des langues"

Ce n'est pas une question de parler les langues, ce n'est pas le problème. On peut ne pas parler d'autres langues que la sienne. C'est plutôt la manière même de parler sa 
propre langue, de la parler fermée ou ouverte, de la parler dans l'ignorance de la présence des autres langues ou dans la préscience que les autres langues existent et qu'elles nous influencent même sans qu'on le sache. Ce n'est pas une question de science, de connaissances des langues, c'est une question d'imaginaire des langues. Et, par conséquent, ce n'est pas une question de juxtaposition des langues, mais de leur mise en réseau (Glissant, 2009 : 27-28).

13 Cette caractéristique les amène à revisiter les schémas communicationnels occidentaux véhiculés par l'école, par nature plus normés et académiques.

14 J'ai ainsi pu observer in situ les transpositions des pratiques langagières de la sphère privée vers la sphère professionnelle. À l'oral, au téléphone, les interactions prennent rapidement une tournure conversationnelle, mêlant le professionnel au privé (notamment la famille) en tissant les langues au gré des propos. Dans les courriels qui s'avèrent être une nouvelle forme d'écrit fortement influencé par l'oral, les agents visas cultivent la relation et le sens plutôt que la correction grammaticale.

15 Mon adhérence au terrain qui m'a amenée à être acteur parmi les acteurs, a ainsi contribué à la description du personnage archétype de "passeur de langues" soit (Morel-lab, 2009 : 62) : "un individu capable d'impulser une dynamique de contact entre les langues". Au cours de ma recherche en doctorat, j’ai pu préciser :

L'ensemble de ces pratiques qui relèvent de ce que j'ai nommé après Jean-Pierre Segal et Patrice Godin, "culture de la relation" renvoie aux usages plurilingues développés par les agent-visas, en particulier la relation qu'ils entretiennent avec leurs langues d'appartenance, qu'ils déclarent ne pas maîtriser sur le plan linguistique mais qui leur sont essentielles sur le plan relationnel. (Morel-lab, 2014 : 164)

16 Cette "culture de la relation" est l'un des fondements qui leur permet de mettre en œuvre des principes de coopération reposant sur des valeurs morales de solidarité individuelle et collective opérées par des stratégies informelles culturellement référencées. Cette approche océanienne remet en question la conception occidentale de langues compartimentées.

\section{Le plurilinguisme, un outil de travail}

17 Le plurilinguisme des agents visas "passeurs de langues" qui, tombe sous le coup de la norme dès lors que leurs compétences linguistiques sont évaluées dans une langue donnée selon des grilles d'analyse académiques (y compris celles du Cecrl), est de fait invisible à l'organisation qui, par ailleurs, comptabilise scrupuleusement le nombre des dossiers traités par chaque agent.

Ceci participerait à ce qu'Alexandre Duchêne intitule la "parole d'œuvre", expression d'un "néolibéralisme du plurilinguisme", qui exploiterait le langage comme la "matière première" des nouvelles économies de service.

De fait, les profils professionnels des "passeurs de langues", s'inscrivent bien dans cette perspective. Sur le projet de KNS, les postes "agents visas" sont référencés dans la catégorie "Secrétariat et assistance" recouvrant les postes les moins qualifiés de la section "Support à l'entreprise" du Rome ${ }^{6}$, outil qui, en Nouvelle-Calédonie, sert à calculer le degré de protection en matière d'emploi local d'un poste. Les descriptifs de ces postes ouverts prioritairement aux locaux ne font pas mention de compétences plurilingues spécifiques. Ces postes se trouvent ainsi catégorisés parmi les moins considérés et valorisés de l'ensemble des postes administratifs du projet. Leurs conditions salariales au 
moment de leur embauche sont proches de celles des ouvriers sans qualification, confirmant que ces postes du tertiaire sont à la "parole d'œuvre" ce que la main d'œuvre est aux métiers du primaire et du secondaire.

En revanche, sur le chantier de KNS comme à l'aéroport de Zurich, site d'une étude de terrain d'Alexandre Duchêne, les compétences communicationnelles en anglais sont un critère de recrutement implicite :

L'anglais, quant à lui, constitue la langue par défaut qui permet de communiquer avec le plus grand nombre de voyageurs qui ne parleraient pas l'une ou l'autre des langues nationales. En ce sens, l'anglais occupe une place prépondérante pour les professions visibles et semi visibles, faisant l'objet d'une attention soutenue dans les processus de recrutement. Sa dimension non marquée, permettant d'interagir avec une population internationale, suit donc une logique pragmatique destinée à répondre aux attentes du plus grand nombre (Duchêne, 2011 : 81-108).

De fait, la dimension pragmatique de l'anglais est tout à fait prépondérante dans le cadre des grands projets internationaux qui, par nature, se situent dans la globalisation et, par essence, se développent selon des modèles organisationnels anglo-américains avec une large priorité donnée à l'anglais international. Dans cette logique, la maîtrise de l'anglais parlé et écrit est l'un des prérequis dans le recrutement du personnel administratif. $\mathrm{Ce}$ critère privilégie indirectement les anglophones et contribue à accentuer en interne les rapports de force entre les langues.

En Nouvelle-Calédonie, territoire insulaire francophone d'un Pacifique sud majoritairement anglophone, cette logique est renforcée du fait de la rareté des candidats locaux anglophones. Les services de placement calédoniens ne disposent pas dans leurs fichiers, de profils associant un niveau d'étude intermédiaire à un niveau d'anglais élevé. C'est ainsi que l'anglais requis pour les postes les moins qualifiés de la section "Support à l'entreprise" est évalué selon des critères assez flous. Concrètement, ils se limitent à quelques échanges conversationnels en anglais et, le cas échéant, à un test écrit de quelques phrases. Le paradoxe tient dans le fait que ce capital nécessaire pour être considéré lors d'un recrutement et qui constitue donc une sorte de plus par rapport à d'autres candidats locaux qui eux ne peuvent répondre à ce prérequis, n'est plus valorisé à compter de la prise de poste. Dans les faits, l'anglais, langue de communication du projet, est considéré comme allant de soi dans la tenue d'un poste administratif quel que soit son niveau de qualification. En outre, lors de ces recrutements, aucune attention particulière n'est portée sur les langues locales des candidats. En réalité, les compétences plurilingues liées aux appartenances locales sont le plus souvent ignorées voire dans bien des cas insoupçonnés. Quand elles sont connues, elles sont alors considérées comme un fait naturel faisant partie d'une identité propre n'ayant pas d'intérêt sur le plan professionnel puisque ces langues ne disposent d'aucune valeur économique sur le marché linguistique occidental.

\section{Parole d'œuvre et œuvre de parole}

Dans cette troisième partie, je m'attacherai à montrer comment la non reconnaissance des langues locales en milieu professionnel ne permet pas la prise en compte des compétences plurilingues des acteurs de terrain contribuant ainsi à renforcer les phénomènes de ségrégation professionnelle. 
Sur le projet de KNS, les langues locales ne sont pas répertoriées, elles n'ont donc pas d'existence officielle. Associées aux relations privées entre personnes d'une même origine, elles sont le plus souvent considérées comme exotiques par les occidentaux. Cette vision postcoloniale de la valeur des langues est contredite par mon observation in situ qui met en lumière les savoir-faire des "passeurs de langues". En effet, ceux-ci ne s'acquièrent pas en situation d'enseignement, en tout cas pas au sein de l'institution scolaire française qui peine à la prise en compte des langues et cultures minoritaires (Dinvaut, 2013), particulièrement en contexte colonial comme le rappellent les auteurs en préface de l'ouvrage collectif Vers une école plurilingue dans les collectivités françaises d'Océanie et de Guyane :

Le modèle national manifeste une tolérance très relative à la promotion des autres langues que le français à l'école primaire. L'école républicaine avait même pour objectif de faire disparaître les particularismes culturels et linguistiques locaux. Promouvoir les langues locales et la diversité linguistique et culturelle peut donc paraître contradictoire avec le projet initial de l'école: cela reviendrait à compromettre le processus d'homogénéisation dont elle est l'un des principaux instruments. . Notons que la contribution de Marie Salaün à cet ouvrage, modalise en précisant : "On a tendance à se représenter une colonisation de type britannique plus souple, plus pragmatique, plus respectueuse des cultures locales, contre une rigidité républicaine française assimilationniste et négatrice du fait indigène. En réalité, à la lumière des témoignages des élèves, donc du terrain, on réalise que le modèle de l'administration indirecte et celui de l'administration directe ont finalement plus en commun qu'on ne veut bien le dire, ce qui montre qu'on est surtout face à des idéaux-types qui ne peuvent être analysés qu'à l'aune des pratiques. (Vernaudon, Fillol, 2009:6)

En revanche, l'accompagnement mis en place au sein du service a eu pour effet de permettre à ces jeunes océaniens de prendre confiance dans leurs propres savoirs. Ceci a contribué à valoriser leur estime d'eux-mêmes. Chaque nouvelle interaction nécessaire à la réalisation de leurs tâches administratives est ainsi devenue l'occasion de combiner de nouvelles connections entre des pratiques professionnelles régies par des règles occidentales à visée universalistes et des pratiques plus personnelles marquées par une vision du monde océanienne.

Mon adhérence au terrain m'a permis de comprendre comment des savoirs expérienciels s'agrègent, au fil du temps et dans l'action, à des savoirs plus normés. Impalpables, ces savoirs expérienciels mêlent compétences relationnelles, interculturelles et communicationnelles. Ils constituent une "œuvre de parole" dépendante de la capacité des acteurs océaniens à transposer dans leurs pratiques professionnelles, les savoirs informels acquis du fait des pratiques langagières qu'ils entretiennent dans leur sphère privée.

27 Cette "œuvre de parole", continuum qui fait fi des frontières linguistiques, constitue la part langagière de leur travail. Elle contribue à les rendre performants dans la tenue de leur poste en leur permettant d'accomplir leurs tâches administratives dans des délais compatibles avec les besoins du projet. En outre, son invisibilité statutaire est renforcée du fait qu'elle n'apparait pas dans les profils de poste d'agent-visa et que de plus, elle n'est pas mesurable selon les critères d'évaluations académiques. Cette réalité est particulièrement palpable sur un chantier de construction dont l'activité principale est concentrée autour de la gestion de la main d'œuvre.

28 En effet, ce n'est pas le moindre des paradoxes de la mondialisation d'opposer à l'accroissement des mobilités professionnelles, un contrôle de plus en plus strict de la 
circulation des personnes. La contradiction de cette dynamique contribue fortement à la lourdeur des procédures administratives nécessaires au déplacement de la main d'œuvre primordiale pour la progression des grands chantiers.

Sur le projet de KNS, la main d'œuvre indispensable aux travaux n'aurait pu être déplacée sans la contribution d'une autre force de travail, "la parole d'œeuvre" des "passeurs de langues", laquelle reste néanmoins subordonnée aux activités de construction. Comme l'indique l'intitulé "service support" attribué au CCV dans la carte organisationnelle du projet, la réalisation des tâches des "passeurs de langues" est considérée comme non productive au sens restreint du terme puisqu'elle ne génère pas de profit comptable direct. En outre, elle est liée à la quantité de main d'œuvre présente sur le site. C'est ainsi que le nombre des agents visas a fluctué au cours des années du projet. A ce propos, je rappelle que l'avancement du chantier est estimé à partir des quantités de matériaux utilisés et du nombre d'heures de travail nécessaires à la réalisation des ouvrages. Sur le plan comptable, la part des activités de services liée à cette production est estimée selon des pourcentages prédéfinis. Elle est calculée par les logiciels de gestion de projet au prorata du type d'activités. La répartition par tâche induite par ce mode de gestion, afin de mieux en contrôler la productivité, plus difficile à quantifier que dans les activités de main d'œuvre, ne tient aucun compte des nombreuses différences dans le traitement des dossiers qui, selon la nationalité des ressortissants, leur lieu de résidence, la nature et la durée prévue de leur séjour sur le chantier peuvent être plus ou moins compliqués à instruire. En tout état de cause, le mode de calcul qui lie la productivité au nombre des dossiers traités ne permet pas la prise en compte des compétences transversales complexes mises en œuvre par les "passeurs de langues" dans la réalisation de leurs tâches.

C'est ainsi que leur contribution active à la parole d'œuvre du projet reste évaluée selon des critères purement quantitatifs (le nombre de dossiers traités par jour, par mois, etc.) suivant en cela une logique tayloriste dans l'organisation du travail. Par nature, ces critères ne peuvent prendre en compte le savoir-faire expérienciel des "passeurs de langues" et donc la valeur de leur "œuvre de parole". A titre d'exemple, je ferai référence aux messages personnalisés adressés à l'équipe à l'occasion d'un milestone ${ }^{7}$ (le dépôt du $1000^{0^{m} \mathrm{e}}$ dossier). Cette gratification purement honorifique attestait de l'impossibilité structurelle de l'organisation à pouvoir apprécier la valeur du travail fourni pour atteindre ce but. En outre, si son capital symbolique a eu un effet valorisant pour les "passeurs de langues" qui se sont sentis reconnus comme faisant partie d'un collectif œuvrant pour le développement de la Nouvelle-Calédonie, ce message de félicitations ne constituait pas une reconnaissance de leur "œuvre de parole", qui non quantifiable, demeure ignorée par l'organisation. De ce fait, l'"œuvre de parole" des "passeurs de langues" ne donne pas lieu à une valorisation formelle qui pourrait contribuer à leur évolution professionnelle.

\section{Vers une didactique de l'action}

Dans cette dernière partie, l'"œuvre de parole" ne sera plus examinée du point de vue de l'organisation qui l'exploite en la transformant en "parole d'œeuvre" mais du point de vue des acteurs eux-mêmes. En effet, la diversité des cas individuels traités par les "passeurs de langues" dans leurs activités quotidiennes d'agents visas et la nécessité pragmatique d'avoir à les résoudre les ont motivés à améliorer leurs compétences langagières en 
français et en anglais, les deux langues de communication du projet. De plus, le formalisme des démarches à entreprendre tant auprès des services consulaires que de l'administration de la Nouvelle-Calédonie leur a rapidement fait comprendre l'intérêt de maîtriser le langage normé. En tant que formatrice, j'ai eu à accompagner ces processus d'apprentissages basés sur le retour d'expérience. J'ai ainsi pu observer comment, en se frottant aux effets concrets de leurs interactions, ils ont peu à peu transformé les outils mis à leur disposition et ont appris à réinvestir les acquis d'une série d'interactions dans une nouvelle série d'interactions quand bien même celle-ci n'était pas identique à la précédente. De ce fait, les actes de langage de leurs échanges ont pris une valeur formative. En définitive, la particularité de leur approche non conventionnelle, basée sur leurs savoirs expérienciels du plurilinguisme, s'est avérée dynamique, leur permettant d'améliorer leurs connaissances selon des modalités qui échappent aux modèles classiques de la didactique des langues y compris ceux instaurés par le Cecrl.

L'observation in situ sur une période longue m'a ainsi permis d'observer la mise en œuvre d'une "didactique de l'action" caractérisée par une didactique qui s'opère en situation au travers des actes (perspective actionnelle du Cecrl) et de la prise de conscience par l'apprenant de ses apprentissages. De ce fait, elle intègre des dimensions propres à la socio-didactique et d'autres issus de l'ergologie (Dinvaut, 2013). En situation, ceci correspond à la manière dont ces agents visas ont su utiliser les artefacts mis à leur disposition pour améliorer leurs compétences communicationnelles et relationnelles. En adaptant ces outils à vocation professionnelle, ils ont transformé leurs situations de travail en "situations didactiques" (Rispail, 2003). Parallèlement, ces situations leur ont permis de prendre conscience de leur potentiel d'amélioration. Ceci est confirmé par le fait que lors de la mise en place du plan de formation professionnelle en 2011, l'équipe a demandé à pouvoir suivre une formation en anglais. Une formatrice s'est déplacée sur le lieu de travail à raison de 2 heures par semaine pendant 2 mois pour travailler avec l'ensemble de l'équipe réunie pour la circonstance. Cette passerelle entre expérience de terrain et formation professionnelle s'est avérée bénéfique tant sur le plan linguistique que sur le plan de l'estime de soi. Participant à un processus informel de reconnaissance des compétences acquises en situation, elle a contribué à la mise en place d'une spirale bienfaisante qui leur a permis de retrouver le goût des apprentissages plus académiques et de renouer les liens brisés par "l'école indigène".

\section{Conclusion}

La recherche menée sur le terrain d'étude des phases de construction des grands projets miniers en Nouvelle-Calédonie a permis de mettre en lumière l'œuvre de parole des "passeurs de langues", agent-visas océaniens employés au CCV, interface structurelle entre les dynamiques de développement local et les enjeux internationaux liés à l'économie mondialisée. J'ai relié cette compétence à leurs savoirs expérienciels du plurilinguisme en contexte diglossique et à leur culture de la Relation. Ceci m'a amenée à examiner les transformations en cours dans le monde du travail, en particulier les conséquences de sa tertiarisation. J'ai ainsi établi le constat que bien qu'essentielle aux organisations qui l'exploitent, l'œuvre de parole de ces "passeurs de langues" ne bénéficie d'aucune reconnaissance professionnelle participant de ce fait à la constitution d'une parole d'œuvre. En revanche, sur un plan plus individuel, j'ai pu observer comment cette "œuvre de parole" contribue à la mise en œuvre d'une didactique de l'action qui, à 
l'expérience de terrain, associe la prise de conscience par les acteurs eux-mêmes de leur potentiel plurilingue. Cette dernière observation pourrait constituer une ouverture en matière de didactique des langues en milieu professionnel en favorisant une meilleure prise en compte des compétences transversales développées par des individus plurilingues employés à la réalisation de tâches administratives dans les activités du tertiaire.

\section{BIBLIOGRAPHIE}

Boutet, J. (1991). "Activité de catégorisation sociale dans la parole ordinaire". Langage et travail, n $\circ 1$.

Boutet, J. \& Heller, M. (2007). "Enjeux sociaux de la sociolinguistique : pour une sociolinguistique critique". Langage et société, $\mathrm{n}^{\circ} 121-122$. pp. 305-318.

Canut, C. \& Caubet, D. (2002). Comment les langues se mélangent, Codeswitching en Francophonie. Paris : L'Harmattan.

Canut, C. \& Duchêne, A. (2011). "Introduction. Instrumentalisations politiques et économiques des langues : le plurilinguisme en question". Langage et société, n 136. pp. 5-12.

Calvet, L-J. (2001). Linguistique et colonialisme. Paris : Petite Bibliothèque Payot.

Calvet, L-J. (2005) "Mondialisation, langues et politiques linguistiques -Le versant linguistique de la mondialisation ". Disponible en ligne http://ressources-cla.univ-fcomte.fr/gerflint/Chili1/

Calvet.pdf.

Colombel, C. (2011). "Quel espace possible pour les langues océaniennes avec le français dans la société calédonienne ?". Travaux de didactique du français langue étrangère, n 63. pp. 115-130.

Cummins, J. (2013). "L'éducation bilingue : qu'avons-nous appris de cinquante ans de recherche ?".In Nocus, I., Vernaudon, J. \& Paia, M. (dir.). Apprendre plusieurs langues, plusieurs langues pour apprendre. L'école plurilingue en Outre-mer. Rennes : Presses Universitaires de Rennes, Collection Des sociétés . pp. 41-65.

Dabene, M. \& Rispail, M. (2008). "La sociodidactique : naissance et développement d'un courant au sein de la didactique du français en France". Lettre de l'AIRDF, n 42. pp. 10-13.

Dinvaut, A. (2013). "Penser de concert l'ergologie, la sociolinguistique, la sociodidactique".

Ergologia, $\mathrm{n}^{\circ}$ 8. pp. 23-59

Dinvaut, A. (2011). "Une approche ergologique des activités langagières et didactiques". Les recherches-actions en Asie du sud-est et ailleurs! Synergies Gerflint. pp. 153-164.

Duchêne, A. (2011). "Néolibéralisme, inégalités sociales et plurilinguisme : l'exploitation des ressources langagières et des locuteurs". Langage et Société, n 136. pp. 81-106.

Duchêne, A. \& Heller, M. (2012). "Multilingualism and the new economy". In Martin-Jones, M., Blackledge, A. \& Creese, A. (eds.) The Routledge Handbook of Multlilingualism. New York: Routledge. pp. 369-383. 
Duchêne, A. \& Heller, M. (2012). Language in late capitalism. New-York: Routledge.

Fiscetti, F. (2009). Théories du multiculturalisme, un parcours entre philosophie et sciences sociales. Paris : La découverte/M.A.U.S.S.

Fillol, V. (2013). "Pour une sociodidactique des langues et des cultures : enjeux, démarches, éthique. Une expérience océanienne". Synthèse d'HDR, Université Jean Monnet, Saint-Etienne.

Glissant, E. (1990). Poétique de la Relation. Paris : Gallimard.

Glissant, E. (2009). L'imaginaire des langues. Paris : Gallimard.

Glissant, E. (2009). "Culture et colonisation : l'équilibre antillais". Disponible en ligne http:// socio13.wordpress.com/2009/02/25/culture-et-colonisation-lequilibre-antillais-par-edouardglissant/

Godin, P. (1998). "L’enfant silencieux : Une éducation kanak dans un monde en changement". In Ihage, W. (dir). Actes du $10^{\text {ème }}$ colloque Corail "Education, culture et identité". Nouméa.

Gorode, D. (1995). "De l'usage du français et des langues kanak", Mwa Véé. n 9, Nouméa : ADCK. pp 23-26.

Gumperz, J.J. (1989). Sociolinguistique interactionnelle, une approche interprétative. Paris :

L'Harmattan.

Heller, M. \& Boutet, J. (2006). "Vers de nouvelles formes de pouvoir langagier : langue(s) et identité dans la nouvelle économie" Langage et société, n 118. pp. 5-16.

Kerbrat-Orecchioni, C. (1999). Les interactions verbales, Approche interactionnelle et structure des conversations. Paris: Armand Colin, tome 1.

Lavric, E. (2006). "Theses About Business Languages Choices" Conference on Multilingualism and Applied Comparative linguistics, Brussels.

Mari, I. (2006). "Multilinguism in companies in the worldwide market: Using the British strategy as an example". Noves SL. Revista de Sociolingüística

Moïse, C. (2009). "Pour une sociolinguistique ethnographique. Sujet, discours et interactions dans un espace mondialisé". Synthèse d'HDR, Université François Rabelais, Tours.

Mokaddem, H. (2011). L'Accord de Nouméa pour tous, Nouméa : Publications de l'IFMNC.

Morel-lab, A. (2008). "L'impalpable au travail Contacts de langues en milieu industriel multilingue Etude contextualisée dans le cadre du projet Goro-Nickel en Nouvelle-Calédonie". Mémoire de Master 1, Université Stendhal, Grenoble.

Morel-lab, A. (2009). "L'impalpable au travail : les interférences de langues dans les relations professionnelles. Quelle langue pour quel usage? Etude contextualisée dans le cadre du projet ValeInco en Nouvelle-Calédonie". Mémoire de Master 2, Université de la Nouvelle-Calédonie, Nouméa.

Morel-lab, A. (2014). "Les passeurs de langues, acteurs de l'interculturel en milieu professionnel plurilingue, le cas des grands projets miniers en Nouvelle-Calédonie". Thèse, Université de la NouvelleCalédonie, Nouméa.

Morin, E. (2000). Les sept savoirs nécessaires à l'éducation du futur, Paris : Seuil.

Nocus, I., Vernaudon, J. \& Paia, M. (éd.) L'école plurilingue outre-mer, apprendre plusieurs langues, plusieurs langues pour apprendre, Rennes : Presses universitaires de Rennes.

Rispail, M. (2003). Le francique : de l'étude d'une langue minorée à une sociodidactique des langues, Paris : L'Harmattan. 
Salaün, M. (2005). L'école indigène. Nouvelle-Calédonie. 1885-1945. Rennes : Presses universitaires de Rennes.

Salaün, M. (2013). Décoloniser l'école ? Hawai'i, Nouvelle-Calédonie. Expériences contemporaines. Rennes : Presses Universitaires de Rennes, collection Essais.

Segal, J-P. (2009). Le Monde du travail au cœur du destin commun, Nouméa : Direction du Travail et de l'Emploi Nouvelle-Calédonie.

Thamin, N. (2007). Dynamique des répertoires langagiers et identités plurilingues de sujets en situation de mobilité, Thèse de doctorat, Université Stendhal, Grenoble.

Vergnaud, G. (1996). Au fond de l'action, la conceptualisation. Savoirs théoriques et savoirs d'action. Paris : Presses universitaires de France.

Vernaudon, J. \& Fillol, V. (2009). Vers une école plurilingue dans les collectivités françaises d'Océanie et de Guyane. Paris : L'Harmattan.

\section{NOTES}

1. Koniambo Nickel SA est une entreprise minière dont les Kanak sont propriétaires à $51 \%$ au travers de la société d'économie mixte Sofinor appartenant à la Province Nord de la NouvelleCalédonie. Sa constitution a été au cœur des discussions lors de la signature du Protocole de Bercy, un accord sur le nickel, préalable à la signature de l'accord de Nouméa.

2. Bien qu'institutionnellement reconnu, le statut des langues d'appartenance de la NouvelleCalédonie reste tout à fait minoritaire y compris dans le cadre des programmes d'enseignement à l'école primaire.

3. Voir sur le site du projet http://www.dylan-project.org, l'ensemble des travaux de recherche menés au cours de la période dans «l'optique de la création d'une société fondée sur la connaissance (...) Son but a été de décrire en quoi différents modes de penser, d'argumenter et d'agir, inhérents aux différentes langues, contribuent à la construction et au transfert des connaissances et interviennent dans le contrôle de l'interaction, la résolution de problèmes et la prise de décision. Le projet visait à fonder scientifiquement la construction de répertoires plurilingues comme ressources pouvant être mises en œuvre dans la diversité des contextes professionnels, politiques et éducatifs "

4. Le Centre de développement de l'ocde (Organisation de Coopération et de Développement Economiques) rappelle en introduction de la publication Repères $n^{\circ} 69$ datée de juin 2008 que ces phénomènes se sont largement amplifiés au cours des trente dernières années.

5. Le réseau pluridisciplinaire Langage et Travail a été créé en 1986. 9 cahiers Langage et Travail ont été publiés entre février 1991 et septembre 1997 téléchargeables sur le site du réseau : http:// www.langage.travail.crg.polytechnique.fr

6. Le Répertoire Opérationnel des Métiers et des Emplois est l'outil de référence français en matière d'emploi. Les multiples activités professionnelles des grands projets ont été référencés à ce code suite à un important travail de transposition entre les logiques organisationnelles propres aux entreprises internationales et les prérequis de la législation en matière d'emploi local applicable en Nouvelle-Calédonie.

7. Jalon en français. Ce mot fait partie du jargon professionnel en gestion de projet. Dans la logique quantitativiste, il définit un objectif de résultat. Dans le cas du CCV, il s'agit du nombre de dossiers traités. 


\section{RÉSUMÉS}

La recherche en ethnographie linguistique menée sur le terrain d'étude des phases de construction des grands projets miniers en Nouvelle-Calédonie, a permis de mettre en lumière la valeur d'un plurilinguisme non normé, ancré dans des langues d'appartenance sans valeur marchande. Ce plurilinguisme de la Relation, dont la didactique est intrinsèquement liée à l'activité professionnelle, est celui des "passeurs de langues", agents administratifs d'un service dédié à la mobilité internationale des travailleurs de ces grands chantiers. Participant pleinement à la tertiarisation d'une main d'œuvre dont le capital linguistique ne constitue pas un capital économique, cette "didactique de l'action" questionne les pratiques d'acquisition et de transmission des langues.

Ethnolinguistic research led during the construction phases of the big mining projects in New Caledonia, allowed to bring to light the value of a non-standardized plurilingualism, anchored in languages with no economic value. This plurilingualism of the Relation and its didactics which is instrinsically connected to professional activity is the one of "langage go-betweens", administrative assistants of a department dedicated to international mobility of the workforce involved in these big construction projects. As part of the transformation processes of the workforce into a "wordforce", the linguistic capital of these "langage go-betweens" does not lead them to better professional recognition. However, this "didactics of action" questions languages acquisition and transmission practices.

\section{INDEX}

Mots-clés : plurilinguisme, capital linguistique, interculturel, multiculturalisme, didactique Keywords : plurilingualism, linguistic capital, intercultural, multiculturalism, didactics

\section{AUTEUR}

\section{ANNE MOREL-LAB}

Anne Morel-Lab a soutenu sa thèse en anthropologie sociale et sciences du langage à l'Université de la Nouvelle-Calédonie en mars 2014. Elle y développe une approche pluridisciplinaire pour étudier les rapports de langues en situation professionnelle sur un terrain d'étude spécifique : les phases de construction des grands projets miniers en Nouvelle-Calédonie, par ailleurs lieu d'exercice de son activité professionnelle de formatrice. De retour en métropole depuis quelques mois, elle cherche à poursuivre sa démarche de recherche sur les rapports de langues en situation professionnelle pour identifier les impacts des nouveaux modes de production dans un monde du travail globalisé.

Courriel : annemorellab@gmail.com.

Adresse : 26, rue Jules Migonney 01000 Bourg-en-Bresse - France 